\title{
PROYECTO DE UN SISTEMA LIDAR TROPOSFÉRICO EN COCHABAMBA - BOLIVIA
}

Omar Ormachea, Oscar Urquidi, Oscar Claure, Alejandro Durandal, Joseth Yavé, Gonzalo Conde y Andrea Cruz

\section{RESUMEN}

En el presente artículo se presenta los avances en la construcción de un sistema LIDAR monostático coaxial para el estudio de las partículas contaminantes existentes en la capa troposférica sobre la ciudad de Cochabamba - Bolivia y que son producto de la actividad agraria en regiones aledañas, muy intensas principalmente en época seca de poca lluvia y que empañan completamente el cielo. El proceso de construcción de este sistema ha incluido el diseño, ajuste y prueba de la óptica de envío y recolección de la luz láser retrodispersada. El sistema usa dos láseres continuos semiconductores de longitudes de onda $\lambda=655 \mathrm{~nm}$ y $\lambda=532 \mathrm{~nm}$, un telescopio newtoniano de $114 \mathrm{~mm}$ de diámetro y 1 metro de distancia focal y una cámara digital Canon Xsi como sensor óptico.

Palabras Clave: Láseres, Opto-Electrónica, Espectroscopia. 